\title{
Adaptive Path Scheduling for Mobile Element to Prolong the Lifetime of Wireless Sensor Networks *
}

\author{
Dakai Zhu and Ali Şaman Tosun \\ University of Texas at San Antonio \\ San Antonio, TX 78249 \\ \{dzhu,tosun\}@cs.utsa.edu
}

\begin{abstract}
Mobile elements, which can traverse the deployment area and convey the observed data from static sensor nodes to a base station, has been introduced for energy efficient data collection in wireless sensor networks (WSNs). However, most existing solutions only calculate a single path for the mobile element, which may lead to quick energy depletion for sensor nodes that are far away from the path. In this paper, for real-time data collection in a WSN with one mobile element, we study the adaptive path scheduling problem for prolonging the lifetime of the WSN. Here, multiple paths are planned and the mobile element follows the paths in turn to balance the energy consumption on individual sensor nodes, thus to extend the WSN's lifetime. We first illustrate the problem with one motivational example. Then, for cases where the movement of the mobile element is restricted (e.g., straight lines), we propose and analyze the optimal solutions. For the general cases, we discuss the issues involved and speculate our future research directions.
\end{abstract}

\section{Introduction}

In the recent past, the popularity of wireless sensor networks (WSNs) has been manifested by their deployment in many real-life applications (e.g., habitat study [4] and ecology monitoring [7]). With potentially a large number of sensor nodes scattered in a region of interest, the main problem in WSNs is how to efficiently aggregate the data at each node to a base station, which has the computational power to store and process all the collected data $[1,2]$. Note that, sensor nodes are generally battery powered and it is hard (if not impossible) to replace those batteries after their deployment. Therefore, developing energy efficient data collection schemes is ultimately important.

In conventional WSN deployments, the data collection

* This work was supported in part by NSF awards CNS-0720651 and CCF-0702728. is normally achieved by using a multi-hop data forwarding mechanism. Here, for the nodes that are far away and cannot reach the base station in a single hop, the data will be relayed by the near to base station neighbors [1]. However, in this scheme, the energy budget for the nodes that are close to the base station will be quickly depleted due to their high data transmission activities and the lifetime of the WSN is rather limited.

To address this problem, mobile elements, which can move around the deployed field and convey the data from each sensor node to the base station, have been proposed [5, $6,10]$. The main problem in this scheme is how to control the mobility of the mobile elements for efficient data collection while satisfying various constraints (e.g., before buffer is full on each sensor node [6]). More recently, considering the constraint that the mobile element may not be reachable from every sensor node, the hybrid approaches that combine multi-hop and mobile elements have been studied $[3,9,8]$. Here, the data is first aggregated locally using multi-hop schemes to some rendezvous points. Then, the mobile element visits these points to pick the data up [9].

Note that, in the existing studies involving mobile elements, only a single path is calculated for each mobile element and the same path is followed repeatedly during data collection [3, 9]. However, such a solution with a single path may lead to uneven energy depletion rate for sensor nodes in WSNs. For instance, in WSNs where the mobile element collects data from each node directly, the nodes that are far away from the path will use up their energy budget quickly leading to limited lifetime for such WSNs.

In this paper, for real-time data collection in WSNs with a single mobile element that collects data directly from each sensor node, we study the adaptive path scheduling problem. Different from the single-path solutions, the key idea is to calculate multiple paths for the mobile element. During data collection, the paths are followed in turn to balance the energy consumption on individual sensor nodes, thus to extend the lifetime of the WSNs. 


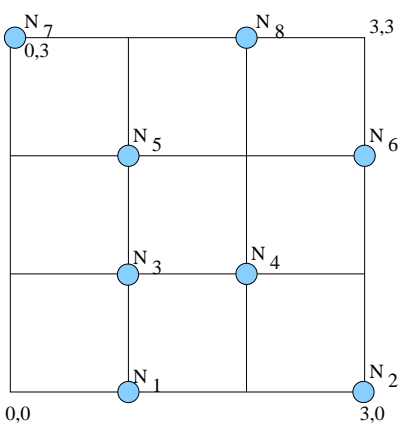

(a) $4 \times 4$ field with 8 sensors

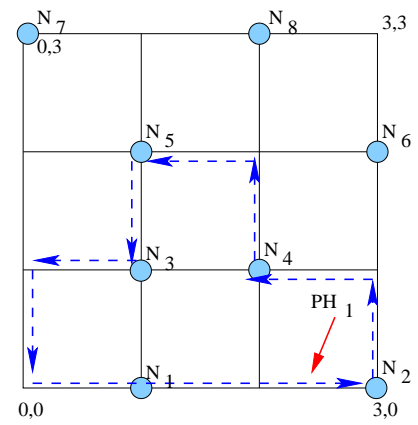

(b) Single path

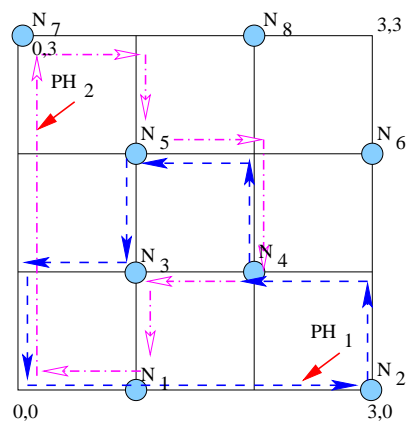

(c) Adaptation with two paths

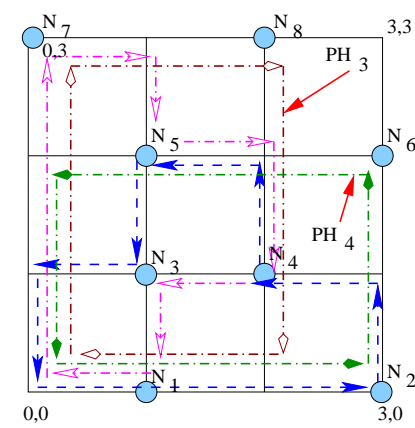

(d) Adaptation with four paths

Figure 1. Motivational Example: Adaptive Paths for one Mobile Element

\section{System Models and Assumptions}

In this section, we first present the system models and state our assumptions. The WSN considered consists of $n$ static sensor nodes that are deployed in the field, one base station and one mobile element. The position for the node $N_{i}(i=1, \ldots, n)$ is given as $\left(x_{i}, y_{i}\right)$, which is assumed to be known. The base station is located at $\left(x_{0}, y_{0}\right)$. Departing from the base station, the mobile element needs to travel through the field, collect data from each sensor node directly and return to the base station for conveying the collected data and recharging within a given time ${ }^{1} T$.

It is well-known that, for wireless communication between two nodes with distance $d$, the transmission power $P$ needed can be modeled as:

$$
P=\alpha d^{\beta}
$$

where $\alpha$ and $\beta$ are system dependent parameters. Suppose that the mobile element follows a travel path $P H$ during one round of data collection, the amount of energy consumed by node $N_{i}$ for transmitting data to the mobile element can be calculated as $E_{i}=P_{i} \cdot t=\alpha d_{i}^{\beta} t$, where $d_{i}$ is the shortest distance from $N_{i}$ to $P H$ and $t$ is transmission time. Assuming that the sensor nodes have the same sampling rate, the amount of data collected at each node will be the same during any time interval $T$ and $t$ will be a constant. The maximum transmission range at the maximum power level $P^{\max }$ is assumed to be $d^{\max }$, which limits the maximum distance from any node to the path of the mobile element.

Therefore, to minimize the energy consumption at each node, it is desired for the mobile element to visit the location of each and every sensor node. However, due to the time limitation $T$, the length of $P H$ will be limited by $L=S \cdot T$, where $S$ is the constant moving speed of the mobile element. Note that the lifetime of WSNs is limited by the node(s) consuming the highest amount of energy.

\footnotetext{
${ }^{1}$ The time may be limited by the buffer size on sensor nodes, or the energy budget of the mobile element.
}

With the goal of maximizing the lifetime of the WSN, in this work, we study the path planning problem for the mobile element. Different from previous work, we focus on adaptive path scheduling, where multiple paths will be planned and are followed in turn by the mobile element to balance the energy consumed at each node.

\section{One Motivational Example}

We first illustrate the problem with one example, where 8 sensor nodes are placed on a $4 \times 4$ grid field as shown in Figure 1(a). Here, the base station is located at $(0,0)$ and the mobile element needs to follow the grid on the field. Suppose that the grid size is 1 and the path length limit of the mobile element is 10 . It can be easily seen that it is not possible for the mobile element to visit each and every sensor node during one round of data collection.

\begin{tabular}{|c|c|c|c|c|}
\hline & $P S_{1}$ & $P S_{2}$ & $P S_{3}$ & $P S_{4}$ \\
\hline \hline$N_{1}$ & $0 \beta t$ & $0 \beta t$ & $0 \beta t$ & $0 \beta t$ \\
\hline$N_{2}$ & $0 \beta t$ & $12 \beta t$ & $9 \beta t$ & $8 \beta t$ \\
\hline$N_{3}$ & $0 \beta t$ & $0 \beta t$ & $6 \beta t$ & $8 \beta t$ \\
\hline$N_{4}$ & $0 \beta t$ & $0 \beta t$ & $3 \beta t$ & $4 \beta t$ \\
\hline$N_{5}$ & $0 \beta t$ & $0 \beta t$ & $3 \beta t$ & $4 \beta t$ \\
\hline$N_{6}$ & $12 \beta t$ & $12 \beta t$ & $9 \beta t$ & $8 \beta t$ \\
\hline$N_{7}$ & $24 \beta t$ & $12 \beta t$ & $9 \beta t$ & $8 \beta t$ \\
\hline$N_{8}$ & $12 \beta t$ & $12 \beta t$ & $9 \beta t$ & $8 \beta t$ \\
\hline Total & $48 \beta t$ & $48 \beta t$ & $48 \beta t$ & $48 \beta t$ \\
\hline
\end{tabular}

Table 1. Energy consumed by the sensor
nodes for transmitting data during 12 rounds
of data collection with different sets of paths.

Suppose that a path $P H_{1}$ is calculated as shown in Figure 1(b). For illustration purpose, we assume that $\alpha=1$ and $\beta=2$. Moreover, the transmission energy for the nodes on the path is assumed to be negligible. For the schemes with the single path $P H_{1}$, after 12 rounds of data collection, the 
energy consumption of each node for transmitting the data is shown in the second column (i.e., labeled as $P S_{1}$ ) in Table 1 . Here, we can see that node $N_{7}$ consumes much more energy than other nodes.

Instead of always following the same path, we may calculate two paths $\left(\mathrm{PH}_{1}\right.$ and $\mathrm{PH}_{2}$, as shown in Figure 1(c)) and the mobile element can follow them alternatively. In this case, the energy consumption of each node for 12 rounds of data collection is shown in the third column (labeled as $P S_{2}$ ) of Table 1 . Suppose that the WSN can be operated until the first node uses up its energy, using two paths can effectively double the lifetime of the WSN as that of the single path option. Note that, the total energy consumed by all the nodes is the same as the previous case.

The lifetime of the WSN can be further improved when more paths can be exploited. The case with four paths is shown in Figure 1(d) and the fourth column of Table 1. Note that the paths may be followed differently by the mobile element for better performance. For the four paths in Figure 1(d), if $\mathrm{PH}_{1}$ and $\mathrm{PH}_{2}$ are followed once while $\mathrm{PH}_{3}$ and $\mathrm{PH}_{4}$ are followed twice in sequence, the corresponding energy consumption of the nodes is shown in the last column in Table 1. Here, the lifetime of the WSN can be tripled compared to that of the single path option. Again, the total energy consumption for all nodes is the same.

Therefore, to maximize the lifetime of the WSN, instead of minimizing the overall energy for all nodes [9], we should focus on minimizing the energy consumption on individual nodes. Another interesting observation from this example is that, for the nodes that are close to the base station (e.g., $N_{1}$ ), their energy consumption is much less since most paths pass by or are close to such nodes.

\section{Adaptive Mobile Element Path Scheduling}

Let's first formally state the adaptive mobile element path scheduling problem with the assumption that the WSN can operate until the first node dies. For a given WSN with $n$ sensor nodes and one mobile element, finding the set of paths $P S=\left\{P H_{1}, \ldots, P H_{k}\right\}$ for the mobile element to:

$$
\operatorname{Minimize}\left(\max _{\forall i} E_{i}=\max _{\forall i} \frac{\sum_{j=1}^{k} \alpha\left(d_{i}^{j}\right)^{\beta}}{k}\right)
$$

subject to

$$
\begin{aligned}
\left|P H_{j}\right| & \leq L, \forall j \\
d_{i}^{j} & \leq d^{\max }, \forall i \forall j
\end{aligned}
$$

where $E_{i}$ is the average energy consumption for node $N_{i}$ for one round of data collection; $\left|P H_{j}\right|$ stands for the length of path $P H_{j}$ and $d_{i}^{j}$ is the minimum distance from node $N_{i}$ to path $\mathrm{PH}_{j} . k$ is the number of paths to be calculated.

\subsection{Restricted Paths}

In some applications, the movement of the mobile element may be restricted [5]. In what follows, suppose that the mobile element can only move horizontally (i.e., in xdirection) along a straight line. We need to find the optimal path location (i.e., y-coordinate) for the mobile element, which will communicate with each node when they are vertically aligned.

For the case of $k=1$ (i.e., a single path is used), $E_{i}$ will reach its maximum value at the sensor node(s) with maximum and/or minimum $y$ coordinates. Therefore, to minimize the maximum value of $E_{i}$, the optimal path location is $Y_{o p t}=\frac{y_{\min }+y_{\max }}{2}$, where $y_{\max }$ and $y_{\min }$ are the maximum and minimum $y$ coordinates of the nodes, respectively. For the case of $k>1$, as stated in the following theorem, the optimal location for all paths will overlap at $Y_{\text {opt }}$. The proof is omitted due to space limitation.

Theorem 1 Suppose that the movement of the mobile element in a WSN is restricted along the x-direction, the location of the optimal path for the mobile element to minimize the highest energy consumption among all nodes is $Y_{\text {opt }}$.

\subsection{Unrestricted Paths}

The problem of finding the general paths is similar to the traveling salesman problem (TSP) with neighborhood and is expected to be NP-hard. In this work, we focus on two different heuristic approaches for solving the problem. Denoted by shrinking path planning (SPP), one approach first constructs the complete path for the TSP which visits all nodes (the computational efficient MST approximation can be used). Then, nodes are removed from the path (with the constraint of Equation 4) one by one until the path length satisfies Equation 3. Starting from the opposite direction, the growing path planning $(G P P)$ approach first finds a partial path by solving the TSP with a subset of nodes. Then the partial path is extended to make sure that the distance from the path to the remaining nodes satisfies Equation 4. If the path is still within the limit, the path can be further extended to reach the remaining node as close as possible.

Focusing on GPP approach, in what follows, we discuss both offline and online heuristic schemes.

Offline Planning for $k$ Paths: To find out $k$ fixed paths offline, we can calculate them independently or iteratively. For the independent scheme, we first divide the $n$ nodes into $k$ seed subsets with each subset having $\left\lceil\frac{n}{k}\right\rceil$ seed nodes in it. This guarantees that each node serves at least as a seed node in one subset. For each of the seed subsets, a path will be calculated following the GPP approach, which will pass by the node in the subset while getting as close as possible to other nodes. 


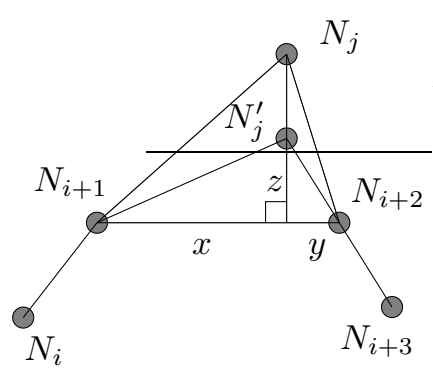

(a) Point on an edge closest

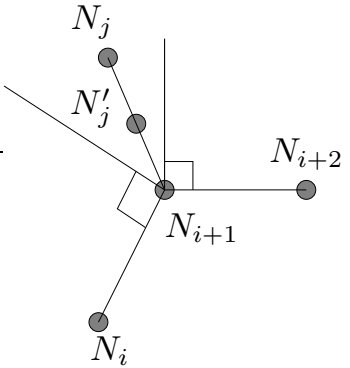

(b) A vertex closest
Figure 2. Addition of a node to a path

For a subset of seed nodes, suppose the initial partial path obtained is $P H_{i}$. The detailed steps for adding a node $N_{j}$ into the path are explained below. Depending on which point on the path $\mathrm{PH}_{i}$ has the minimum distance to $N_{j}$, there are two cases.

The first case is shown in Figure 2(a), where the point is on one edge of $P H_{i}: \ldots, N_{i}, N_{i+1}, N_{i+2}, N_{i+3}, \ldots$ If the distance from $N_{j}$ to the edge $\left(N_{i+1}, N_{i+2}\right)$ is no more than $d^{\max }$, we will ignore the node $N_{j}$ during the first phase of extending $\mathrm{PH}_{i}$. Otherwise, the path has to be first extended to the point $N_{j}^{\prime}$, such that $d\left(N_{j}, N_{j}^{\prime}\right) \leq d^{\max }$. Here, the path length will be increased by $\delta=d\left(N_{i+1}, N_{j}\right)+$ $d\left(N_{j}, N_{i+2}\right)-d\left(N_{i+1}, N_{i+2}\right)$. After extending the path $\mathrm{PH}_{i}$ to node $N_{j}^{\prime}$, the closest point from $P H_{i}$ to node $N_{j}$ can be illustrated as the second case in Figure 2(b).

Suppose that, after incorporating all the remaining nodes in the first phase, the current path length is $\left|P H_{i}\right|$. If $\left|P H_{i}\right|>L$, the construction of $P H_{i}$ fails. Otherwise, during the second phase, we can further extend $P H_{i}$ to get as close to node $N_{j}$ as possible while making sure $\left|P H_{i}\right| \leq L$. In the second phase of extending $P H_{i}$, if $\left|P H_{i}\right|+\delta \leq L$, path $P H_{i}$ can be extended to include node $N_{j}$ by adding edges $\left(N_{i+1}, N_{j}\right)$ and $\left(N_{j}, N_{i+2}\right)$ while removing edge $\left(N_{i+1}, N_{i+2}\right)$. Otherwise, if $\left|P H_{i}\right|+$ $\delta>L$, we can partially add node $N_{j}$ by extending the path to a virtual node $N_{j}^{\prime}$. And the path $P H_{i}$ will be $\ldots, N_{i}, N_{i+1}, N_{j}^{\prime}, N_{i+2}, N_{i+3}, \ldots$ The details on how to calculate the position of $N_{j}^{\prime}$ is omitted due to space limitation. So as the discussion on extending path $P H_{i}$ for the second case shown in Figure 2(b).

For the iterative scheme, we can construct the first path by randomly selecting some seed nodes. After that, the selection of the seed nodes for constructing the $i^{t h}$ path will depend on the energy consumed by the nodes in the first $(i-1)$ paths. Nodes that consumed the highest amount of energy have higher priority for being selected as seed nodes.

Online Adaptive Path Planning/Scheduling: Instead of using $k$ predetermined paths, new paths can be computed on the fly at runtime. Using the same idea in the offline iterative scheme, a new path can be calculated based on the remain- ing energy consumption of the nodes. To amortize the cost of path computation, each path can be used for $R$ rounds of data collection. Or, a new path is calculated whenever the remaining energy ratio between the sensor with most energy and least energy is above a certain threshold $\tau$.

\section{Conclusion and Future Work}

Existing approaches using mobile elements for data collection in WSNs normally plan a single path, which may lead to quick energy depletion for sensor nodes that are far away from the path. In this paper, we introduce the adaptive path planning/scheduling problem, where multiple paths are planned and followed in turn to balance the energy consumption on individual sensor nodes, thus to extend the WSN's lifetime. For cases with restricted movement of the mobile element, one optimal solution is analyzed. For general cases, different approaches to find multiple paths are discussed, where nodes with higher energy consumption are more likely to be on the constructed paths.

For our future work, we will consider cases where the lifetime of WSN can last until multiple nodes die. Moreover, adaptive path scheduling for hybrid schemes with multi-hop data forwarding will be studied.

\section{References}

[1] I. F. Akyildiz, W. Su, Y. Sankarasubramaniam, and E. Cayirci. A survey on sensor networks. IEEE Communications Magazine, 38:393422, 2002.

[2] D. Estrin, R. Govindan, J. S. Heidemann, and S. Kumar. Next century challenges: Scalable coordination in sensor networks. In Mobile Computing and Networking, pages 263-270, 1999.

[3] M. Ma and Y. Yang. Sencar: An energy-efficient data gathering mechanism for large-scale multihop sensor networks. IEEE Trans. on Parallel and Distributed Systems, 18(10):1476-1488, Oct. 2007.

[4] A. Mainwaring, J. Polastre, R. Szewczyk, D. Culler, and J. Anderson. Wireless sensor networks for habitat monitoring. In ACM Int. Workshop on Wireless Sensor Networks and Applications, pages 88 $-97,2002$.

[5] R. C. Shah, S. Roy, S. Jain, and W. Brunette. Data mules: modeling a three-tier architecture for sparse sensor networks. In IEEE SNPA, pages 30-41, 2003.

[6] A. Somasundara, A. Ramamoorthy, and M. Srivastava. Mobile element scheduling for efficient data collection in wireless sensor networks with dynamic deadlines. In IEEE RTSS, pages 296-305, 2004.

[7] G. Tolle, J. Polastre, R. Szewczyk, N. Turner, K. Tu, P. Buonadonna, S. Burgess, D. Gay, W. Hong, T. Dawson, and D. Culler. A macroscope in the redwoods. In ACM SenSys, pages 51-63, 2005.

[8] W. Wang, V. Srinivasan, and K.-C. Chua. Using mobile relays to prolong the lifetime of wireless sensor networks. In ACM MobiCom, pp. 270-283, 2005

[9] G. Xing, T. Wang, Z. Xie, and W. Jia. Rendezvous planning in mobility-assisted wireless sensor networks. In IEEE RTSS, Dec 2007.

[10] Wenrui Zhao, Mostafa Ammar, and Allen Zegura. A message ferrying approach for data delivery in sparse mobile ad hoc networks. In MobiHoc, May 2004. 